\title{
Community care clinicians' experiences and perceptions of waterlow pressure ulcer risk assessment
}

\author{
Heidi A. Green ${ }^{\mathrm{a}, *}$, Janice Wiseman ${ }^{\mathrm{b}}$, Christine Jackson ${ }^{\mathrm{c}}$ \\ a School of Health and Social Care, University of Lincoln, Lincolnshire, United Kingdom \\ ${ }^{\mathrm{b}}$ School of Health and Social Care, University of Lincoln, Lincolnshire, United Kingdom \\ ${ }^{\mathrm{c}}$ School of Health and Social Care, University of Lincoln, Lincolnshire, United Kingdom
}

\section{Introduction}

Pressure ulcers are a complex, aetiologically multifactorial clinical problem [1], that affects approximately 1 in 20 community and 1 in 10 hospital patients [2]. Pressure ulcers are considered to be one of the five most common causes of patient harm [3] that cost the NHS an estimated $£ 1214$ - $£ 40234$ to heal, per episode, depending on pressure ulcer severity and patient health state [4]. Pressure ulcer prevalence is considered a key indicator of care quality, patient safety, and a priority for improvement [5]. Pressure ulcer prevention is a multidisciplinary responsibility with nursing clinicians at the forefront of caregiving [6]. The National Institute for Health and Care Excellence (NICE) recommends the use of a validated assessment framework for pressure ulcer prevention, assessment and management [7]. This guidance identifies ameliorative care pathways for skin care, mechanical loading and equipment resource provision. Therefore, use of a risk assessment tool can be considered as central to guiding pressure ulcer-related care quality [8].

The Waterlow Pressure Ulcer Risk Assessment Tool ('Waterlow') [9] was developed in 1985, for use in medical and surgical settings and is thought to be the most commonly used pressure ulcer risk assessment tool in the UK [10]. Waterlow is used as the tool of choice across the organisation and ameliorative care protocols activate for all patients with a Waterlow score 10 or above i.e. patients scored as being at risk of pressure ulcer development [11]. As such, Waterlow outcome/score informs decisions standardises care practices [12] as well as evidences process [10] of pressure ulcer prevention and management [9]. Waterlow can, therefore, profoundly influence clinicians' actions and how pressure relieving equipment is prescribed [7].

\subsection{Rationale}

To meet the ever-more complex clinical needs of patients and the challenge of the zero pressure ulcer agenda [13], the organisation's assessment, prevention and management care practices were examined. A Tissue Viability Nurse led review, of patient records and Root Cause
Analysis (RCA) investigating pressure ulcers acquired while in the organisations care, highlighted issues of:

- Waterlow completion accuracy;

- Isolated use of Waterlow;

- Waterlow score focused care planning;

- Insufficient prevention care planning;

- Waterlow overestimating risk resulting in:

- Inappropriate equipment prescription;

- Excessive need for ongoing monitoring;

- Risk assessment nursing time when the patient is not professionally judged at risk of pressure ulcer development.

\subsection{Aims}

To explore clinicians' experiences of pressure ulcer, assessment, prevention, and management care practices within the organisation.

\subsection{Ethical considerations}

The work was deemed a service evaluation; it, therefore, did not meet the requirements for formal ethical approval by the Health Research Authority [14,15]. All policies and procedures surrounding research governance and Good Clinical Practice (GCP) were adhered to.

\subsection{Setting}

The service evaluation took place in a Community Health Services NHS Trust within the UK and is referred to as 'the organisation'.

\section{Methods}

\subsection{Terminology}

The term 'survey' is used to denote the data collection method, 'questionnaire' is used to denote the structured series of written

\footnotetext{
* Corresponding author. School of Health and Social Care, University of Lincoln, Sarah Swift, Lincoln, LN6 7TS, United Kingdom.

E-mail addresses: heidi.green1@nhs.net (H.A. Green), janice.wiseman@nhs.net (J. Wiseman), cjackson@lincoln.ac.uk (C. Jackson).
} 
questions administered, i.e. the data collection instrument.

\subsection{Construction of the questionnaire}

The principles described by Dillman [16] were used to devise an electronic survey that was formatted and hosted using an online survey administration website. An anonymously completed, online, largely qualitative questionnaire approach was used to provide the easiest access to clinicians [16], facilitate an opportunity for openness through anonymity [17] and to capture detailed response [18].

A panel of six expert healthcare professionals employed within the organisation supported questionnaire development and its pilot. The panel comprised of two Tissue Viability Clinical Nurse Specialist Leads, a Tissue Viability Clinical Nurse Specialist, a Nurse Specialist, a Research Manager, and a Research Governance specialist. Two of the Clinical Specialists were Community Nurses within the organisation, before undertaking their specialist roles, with one of them moving to their specialist role approximately 1-year before becoming a panel member.

The issues highlighted within in the Tissue Viability Nurse-led review of patient records, and RCA's were used to ground questionnaire development. Three drafts were produced, with each influenced by the feedback from the previous version. Group consensus was used to establish face validity. A pilot of the questionnaire was then undertaken to ensure that questions captured the experience of using Waterlow in clinical practice. No amendments were required.

The final version comprised nine questions and became the Experiences of Using the Waterlow Pressure Ulcer Risk Assessment Tool (EUWT-Q). No personally identifiable data were collected. Demographic data identified professional role and length of time using Waterlow. Questions were both open and closed, however, responses to closed questions asked for response expansion, for example, question 8 asked, "Do you think the Waterlow Pressure Ulcer Risk Assessment Tool effectively guides care planning? Please explain". The last question was designed to elicit free expression by asking "Is there anything you would like to add about your experience of using the Waterlow Pressure Ulcer Risk Assessment Tool?". This final free text response aimed to capitalise on respondents' natural tendency to consider previous answers, encourage the candid expression of opinions not captured within previous questions, and highlight issues of importance [17].

\subsection{Participants}

A Tissue Viability Clinical Nurse Specialist Lead identified a purposive sample of 79 clinicians employed across the organisation in varying roles, that were users of Waterlow within their daily clinical practice. An organisational employed administrator facilitated all contact with participants, no participant information was shared with the researcher.

\subsection{Data analysis}

The analysis adhered to principles of an inductively informed thematic analysis $[19,20]$. Codes were first identified within question-byquestion groups as direct semantic representations, either in vivo, i.e. the code is a verbatim text extract or as a patterned observation, before conceptually driven ideas and assumptions underpinning explicit content were identified. Initial codes were then temporarily disregarded; the data were reread, interconnecting relationships across the dataset were developed and first impressions of themes encapsulating codes established. Initial codes were then compared with secondary codes. Overlapping codes were meaningfully grouped and merged to become overarching concepts across the dataset and were identified as potential theme code groupings. The potential themes code groupings were then explored to identify consistent, inconsistent and divergent content and that they captured the 'essence' of conceptual and explicit content
Table 1

Clinicians characteristic profile.

\begin{tabular}{lllllll}
\hline \multirow{2}{*}{ Job role } & \multicolumn{3}{l}{ Time Using Tool in Years } & \multicolumn{3}{c}{ Total } \\
\cline { 2 - 5 } & $0-5$ & $6-10$ & $11-15$ & $16+$ & \\
\hline Community Nurses (CN) & 5 & 7 & 6 & 20 & 38 & $64 \%$ \\
Nurse Specialists (NS) & 1 & 0 & 1 & 2 & 4 & $7 \%$ \\
Allied Health Professionals (AH) & 4 & 0 & 1 & 0 & 5 & $8 \%$ \\
Healthcare Support Worker (HS) & 7 & 1 & 0 & 0 & 8 & $14 \%$ \\
Other Professions (OP) & 0 & 1 & 0 & 3 & 4 & $7 \%$ \\
Total & 17 & 9 & 8 & 25 & 59 & \\
Percent & $29 \%$ & $15 \%$ & $14 \%$ & $42 \%$ & $100 \%$ & \\
\hline
\end{tabular}

without being over complex or diverse [19,20]. Finally, the potential themes code groupings were refined, defined as themes and supporting verbatim extracts were identified.

\section{Results}

The EUWT-Q yielded rich descriptive responses that included both paragraphical and short contributions. Two interrelated key themes were identified reflect clinicians' experiences of using Waterlow.

\subsection{Respondent characteristics}

Of the 79 clinicians contacted, 59 completed the EUWT-Q (response rate $74 \%)$. Of these, $64 \%(n=38)$ were Community Nurses. Respondents had a high-level of experience using Waterlow with the majority $(42 \% \mathrm{n}=25)$ having used Waterlow for more than 16 years, see Table 1. Clinicians Characteristic Profile.

\subsection{Key code}

Direct quotation extracts are used to illustrate meaning. A key code (Table 2) is used to help identify clinician characteristics. The code is created using three components; a job role code; time using Waterlow; and a unique identification number. For example, clinician CN0562 is a Community Nurse, that has used Waterlow $0-5$ years and is uniquely identified as number 62 .

\subsection{Themes}

\subsubsection{Confidence in waterlow supporting clinical decision-making}

A theme of Confidence in Waterlow Supporting Clinical Decision Making was prominently distinguishable across the dataset. For some, Waterlow was perceived as a "good risk indicator" (CN1620) that helpfully prompted and guided clinical considerations and was a "good baseline tool" (NS0534) that alerted possible patient deterioration. In contrast, for others, completion difficulties emerged. Clinicians expressed confusion with interpreting 'grey areas' and difficulties identifying and classifying risk factors [9]. These difficulties were compounded by the possibility that risk factors could be interpreted differently within their own context, or, differently by different clinicians and as such, had seemingly diminished clinical decision-making confidence as these clinicians explain:

Table 2

Clinician characteristics identification key code.

\begin{tabular}{llll}
\hline Job Role & & \multicolumn{2}{l}{ Time Using Waterlow } \\
\hline CN & Community Nurse & $\mathbf{0 5}$ & $0-5$ years \\
NS & Nurse Specialist & $\mathbf{6 0}$ & 6-10 years \\
AH & Allied Health Professionals & $\mathbf{1 1}$ & $11-15$ years \\
HS & Healthcare Support Worker & $\mathbf{1 6}$ & $16+$ years \\
OP & Other Professions & & \\
\hline
\end{tabular}


"Some sections could be interpreted as ambiguous, it depends on the clinician completing Waterlow as to how they interpret the particular box - especially e.g. under organ failure” (OP1618).

“...it is open to individual interpretation and 2 nurses could assess the same patient and arrive at 2 different scores" (CN1645).

"Lots of phrases around the office by staff saying " would you score ' $x$ ' for neurological? or would you class ' $x$ ' as an organ failure?" (CN6027).

The essence of Waterlow is to predict the risk of PU development [9]. Clinicians considered Waterlow to overestimate risk, and trigger unnecessary or inappropriate clinical facing time and equipment prescription as these clinicians explain:

“...seeing the patient and then seeing their Waterlow score, sometimes do not match up. The patient can be in fairly good health and mobile, yet they can end up with a high Waterlow score meaning they require equipment which isn't always necessary" (HS0549).

“...some patients score high but are fully mobile, but due to high Waterlow score means we have to carry out a SSKINS on each visit which for diabetics this is on a daily basis this can be quite pointless for those patients that are obviously not at risk" (CN1615).

Waterlow was also considered a static tool that was insensitive to health deterioration. Insensitivity was perceived to cause difficulties with process documentation when a patient's health status had changed and their Waterlow score remained static as these clinicians explain:

“...Chronic disease patients have high scores which do not increase significantly when their condition is deteriorating... if a patient has mild oedema, they score the same as severe oedema as there is no variation in scoring available... deterioration in clinical condition means... they need a change in product, frequently would not see any change in the Waterlow score to support this decision" (NS1658).

\subsubsection{Defensively nursing}

The use of Waterlow had seemingly affected the socio-political working culture. Fearful, self-protective care practices were perceived to have evolved in response to organisational imposed ramifications, should patients develop pressure ulcers. This was perceived to have been compounded by a propensity for Waterlow to overestimate pressure ulcer development risk for some patients. Organisational policy was perceived to disempower and overrule clinical judgement, and a pressure to prescribe pressure relieving equipment was reported. This, therefore, had become associated with a working culture that imparted clinical autonomy barriers and defensive patterns of patient care decision-making, where (some), "clinicians focus on the score rather than, foremost, the needs of the patient" (NS1112) had become established. Consequentially, higher-grade equipment was being prescribed, even when the prescription was contradictory to professional judgement.

"It is used as an assessment tool to beat us with as the bar is set to low... We used to use our clinical judgement when doing an assessment but now are too worried about the blame culture that we put in equipment into houses where the patients are fully mobile but have high Waterlow score" (CN6040). "...CCG attitude is geared to blame clinician whatever their choice re-
gardless of comorbid and frailty factors" (CN1637).

“... the zero PU agenda] created an environment of fear which has created a need to over prescribe pressure preventative equipment to those patients scoring high but who do not necessarily require the equipment. This has become acceptable practice to prevent avoidable pressure damage and not what may be best practice for the patient" (CN1635).

A deep frustration was revealed that exposed incongruence between professional philosophy and the clinical actions taken. Clinicians were aware their clinical strategies were counterintuitive and directly linked to defensive nursing, thus they outright requested change:

“...the Waterlow Tool is dated and it is time to look at a tool that is based on more up to date evidence in relation to risk factors. The Waterlow is time-consuming, open to interpretation and does not reliably prioritise the risk factors that are particular to that patient. I believe it is seen as a tick box exercise, another template that HAS to be completed rather than a tool to aid care planning" (NS1112)

"Please replace it as soon as possible" (NS1658).

\section{Discussion}

There is an implicit assumption, that regardless of clinical experience, clinicians will gather the same data, make similar patient care judgements, and that patients with similar needs, follow similar care pathways. It is, however, unreasonable to assume, an assessment outcome will always concur a clinician's professional judgement [21] or, that an assessment outcome replaces experienced clinical evaluation [22]. In the widest sense, this is reflective of practice. Identification of patients who are at high risk of developing pressure ulcers is crucial for determining effective pressure relief management strategies [23,24], and it is the assessment tool that provides the foundational evidence on which to base and standardise these practices [25]. As such, pressure risk assessment tools can be considered to require certain fundamental characteristics. They should correctly identify risk, produce consistent results regardless of the clinician assessing the patient [26] and should appropriately, effectively, and reliably direct pressure relieving equipment resources [27]. It can, therefore, be argued, that clinicians need to perceive the tool they use to assess, prevent, and manage pressure ulcers as valuable, appropriate, and efficacious directing pressure relieving equipment prescription. This is particularly important when considering the influence of the pressure ulcer assessment tool on patterns of care and pressure relieving equipment prescription. Yet, there is no evidence that demonstrates pressure ulcer risk assessment tools adequately identify risk, reduce pressure ulcer incidence or improve patient outcomes [28-32]. As such, clinical judgementis considered superior to pressure ulcer a risk assessment tools for identifying patients at risk of pressure ulcer development [28-32,34].

The findings of this work are supported by the literature that evidence poor inter- and intrarater reliability [31,32], and poor predictive validity of Waterlow [33,34]. These excerpts indicate these clinicians feel little confidence that the use of Waterlow is associated with care practices that are reflective of patient need, or supports clinical decision-making. Thus, over-prescription of pressure relieving equipment may have resulted in some patients receiving inappropriate equipment prescription. DeForge et al. [35] similarly note, how at an organisational level, policy, compliance requirements, risk management expectations and resource pressure can constrain staff and result in tensions that challenge clinician autonomy.

It seems counter-intuitive, that an overprescription of pressure relieving equipment, has not eradicated avoidable pressure ulcers. The solution is, therefore, more complex than one of more pressure preventative resources.

\section{Strengths and limitations}

The EUWT-Q was designed to capture the lived experiences, use of using Waterlow in Clinical practice within the organisation, rather than test a generalisable hypothesis or theory. Therefore, questions were not piloted beyond the Expert Healthcare Professional panel. The EUWT-Q generated rich insight surrounding clinical practice using the organisation's clinicians' own words and meanings. A response rate of $74 \%$, was achieved. Following conventional wisdom, this can be considered a good response rate that procured good data quality [36] thus, 
establishes representativeness and supports the outcomes. The work has provided deeper meaning and wider context to the Tissue Viability Nurse led patient record and RCA reviews that underpin the rationale for the current work. A note of caution is however due. The work was undertaken specifically to inform the organisation, and its clinicians' perceptions and experiences may not be reflective of other clinicians' perceptions and experiences surrounding clinical practice using Waterlow.

\section{Conclusion}

The knowledge produced by the current work has important implications elucidating seemingly costly working practices. Costly in terms of care practices that are not necessarily associated with appropriate pressure relieving equipment prescription and a diminishing of workforce morale. The findings indicate a need for quality improvement innovation and imply the impact of such innovation could be sizable.

\section{Conflicts of interest}

None

\section{Acknowledgement}

The work was funded by a Learning Beyond Registration Fellowship and represents one element of a body of work undertaken and reported for the award of a Masters of Clinical Research.

\section{References}

[1] Briggs M, Collinson M, Wilson L, et al. The prevalence of pain at pressure areas and pressure ulcers in hospitalised patients. BMC Nurs 2013;12:19.

[2] Kaltenthaler E, Whitfield MD, Walters SJ, Akehurst RL, Paisley SUK. USA and Canada: how do their pressure ulcer prevalence and incidence data compare? J Wound Care 2001;10:530-5.

[3] Health and Social Care Information Centre (HSCIC). NHS safety thermometer: patient harms and harm free care england july 2014-july 2015, official statistics. http://www.hscic.gov.uk/thermometer; 2015, Accessed date: 10 September 2015.

[4] Dealey C, Posnett J, Walker A. The cost of pressure ulcers in the United Kingdom. J Wound Care 2012;21:261-6.

[5] Royal College of Nursing (RCN). Pressure ulcers ]. http://www.rcn.org.uk/ development/practice/clinicalguidelines/pressure ulcers; 2015, Accessed date: 16 September 2015

[6] Aslan A, Yavuz van Giersbergen M. Nurses' attitudes towards pressure ulcer prevention in Turkey. J Tissue Viability 2016;25:66-73.

[7] National Institute for Clinical Excellence (NICE). NICE guidelines [CG179] Pressure ulcers: prevention and management of pressure ulcers. http://www.nice.org.uk/ guidance/CG179/chapter/4-The-Guideline-Development-Group-NationalCollaborating-Centre-and-NICE-project-team, Accessed date: 19 September 2015.

[8] Coleman S. The development of a pressure ulcer risk assessment framework and minimum data set [PhD dissertation] Leeds: University of Leeds; 2014.

[9] Waterlow J. Pressure ulcer prevention manual. Taunton: J Waterlow. http://www judy-waterlow.co.uk/the-waterlow-manual.htm; 2005, Accessed date: 7 January
2016.

[10] Guy H. Pressure ulcer risk assessment. Nurs Times 2012;108:16-20.

[11] Trust. SystmOne Champions Group. Trust supporting document for the new holistic and mandatory SystmOne Templates. Author. 2015.

[12] Parsley K, Corrigan P. Quality improvement in healthcare: putting evidence into practice. second ed. Cheltenham: Nelson Thornes Ltd; 1999.

[13] Trust Clinical Strategy (2014/2015). Policy for continuous improvement and innovation. Author. 2014

[14] Health Research Authority (HRA). Defining Research: NRES guidance to help you decide if your project requires review by a Research Ethics Committee. London: HRA; 2013

[15] Health Research Authority (HRA). Is my study research? Decision tool. http:// www.hra-decisiontools.org.uk/research/index.html; 2017, Accessed date: 10 September 2015

[16] Dillman D. Mail and internet surveys: the tailored design method. second ed. New York: John Wiley \& Sons; 2007.

[17] Patten ML. Questionnaire research a practical guide. Los Angeles: Pyrczak Publishing; 1998.

[18] McColl E, Jacoby A, Thomas L, Bamford C, Steen N, Thomas R, et al. Design and use of questionnaires: a review of best practice applicable to surveys of health service staff and patients. Health Technol Assess 2001:5.

[19] Braun V, Clarke V. Using thematic analysis in psychology. Qual Res Psychol 2006;3:77-101.

[20] Braun V, Clarke V. Successful qualitative research: a practical guide for beginners. London: Sage Publications Inc; 2013.

[21] Kapp S. Successful implementation of clinical practice guidelines for pressure risk management in a home nursing setting. J Eval Clin Pract 2013;9:895-901.

[22] van Gilder C, MacFarlane GD, Meyer S. Results of nine international pressure ulcer prevalence questionnaires: 1989 to 2005. Ostomy/Wound Manag 2008;54:40-54.

[23] Kottner J, Dassen T. Pressure ulcer risk assessment in critical care: interrater reliability and validity studies of the Braden and Waterlow scales and subjective ratings in two intensive care units. Int J Nurs Stud 2010;47:671-7.

[24] Keller BP, Wille J, van Ramshorst B, van der Werken C. Pressure ulcers in intensive care patients: a review of risks and prevention. Intensive Care Med 2002;28:1379-88.

[25] Saleh M, Anthony D, Parboteeah S. The impact of pressure ulcer risk assessment on patient outcomes among hospitalised patients. J Clin Nurs 2009;18:1923-9.

[26] Thompson D. Tissue viability. An evaluation of the Waterlow pressure ulcer riskassessment tool. Br J Nurs 2005;14:455-9.

[27] Green HA. Experiences and perceptions using the Waterlow pressure ulcer risk assessment tool: a community care perspective. [Poster] Wounds UK Annual Conference. Harrogate. 2017.

[28] Pancorbo-Hidalgo PL, Garcia-Fernandez FP, Lopez-Medina IM, Alvarez-Nieto C. Risk assessment scales for pressure ulcer prevention: a systematic review. J Adv Nurs 2006;54:94-110.

[29] Nixon J, McGough A. Principles of patient assessment: screening for pressure ulcers and potential risk. In: Morison MJ, editor. The prevention and treatment of pressure ulcers. Edinburgh: Mosby; 2001. p. 55-74.

[30] Vanderwee K, Grypdonck M, Defloor T. Non-blanchable erythema as an indicator for the need for pressure ulcer prevention: a randomized-controlled trial. J Clin Nurs 2007; 16:325-35.

[31] Kottner J, Dassen T. Pressure ulcer risk assessment in critical care: interrater reliability and validity studies of the Braden and Waterlow scales and subjective ratings in two intensive care units. Int J Nurs Stud 2010;47:671-7.

[32] Kottner J, Dassen T, Tannen A. Inter- and intrarater reliability of the Waterlow pressure sore risk scale: a systematic review. Int J Nurs Stud 2009;46:369-79.

[33] Dempsey L, Walsh B. Investigating the reliability and validity of the Waterlow risk assessment scale: a literature review. Clin Nurs Res 2011;20:197-208.

[34] Kottner J, Balze K. Do pressure ulcer risk assessment scales improve clinical practice? J Multidiscip Healthc 2010;3:103-11.

[35] DeForge R, van Wyk P, Hall J, Salmoni A. Afraid to care; unable to care: a critical ethnography within a long-term care home. J Aging Stud 2011;25:415-26.

[36] Babbie ER. Survey research methods. second ed. Belmont USA: Wadsworth; 1990. 